\title{
INVESTIGATION OF FACTORS AFFECTING FREQUENCY OF INTESTINAL PARASITES IN PRIMARY SCHOOL STUDENTS IN AN URBAN REGION IN TURKEY
}

\author{
İnci Arıkan ${ }^{1}$, Aynur Gülcan², Saime Ergen Dıbeklıŏlu ${ }^{3}$ \\ ${ }^{1}$ Department of Public Health, Medicine Faculty, Dumlupinar University, Kutahya, Turkey \\ ${ }^{2}$ Department of Microbiology and Clinical Microbiology, Medicine Faculty, Dumlupinar University, Kutahya, Turkey \\ ${ }^{3}$ Department of Paediatrics, Medicine Faculty, Dumlupinar University, Kutahya, Turkey
}

\section{SUMMARY}

Aim: The aim of the study was to determine the incidence of intestinal parasitic diseases (IPD) and associated factors in primary school students and to assess the knowledge and practices of mothers about these diseases.

Methods: This is a cross-sectional study carried out in January-March 2014 in 471 students aged 5-11 years, studying at 3 schools randomly selected from the city centre regions with different socioeconomic levels. Stratified sampling method was used in the present study and the data were collected in two stages. In the first stage, parents were informed about the study and pre-prepared questionnaire forms were used to collect the data about the students and parents. In the second stage, laboratory analyses of collected stool samples were performed.

Results: The total prevalence of IPD was 18.3\%, it was higher in the primary school located in a region with a lower socioeconomic level compared to other two schools ( $27.6 \%$ vs. $14.4 \%$, and $10 \%$, respectively). Most commonly detected parasite was $E$. vermicularis (12.1\%). The prevalence of IPD was not associated with the classroom, gender, number of siblings, and the use of purified drinking water at home, while it was found to decrease with the increasing maternal education level. The maternal knowledge level score was $12.01 \pm 4.29$ vs. $13.41 \pm 3.94$ in students with and without IPD, respectively. With regard to the methods used to treat IPD, 23\% of the mothers reported that they are using conventional methods.

Conclusion: The health education programmes about the associated risk factors are of great importance for early detection and treatment of childhood parasitic infections.

Key words: intestinal parasitic diseases, prevalence, students, traditional practices

Address for correspondence: A. Gülcan, Dumlupinar University, Medicine Faculty, Department of Microbiology and Clinical Microbiology, 43100 Kutahya, Turkey. E-mail: draynurgulcan@gmail.com

http://dx.doi.org/10.21101/cejph.a4231

\section{INTRODUCTION}

Approximately one quarter of the world population is at risk of getting affected by intestinal parasitic diseases (IPD). It is among the most important health problems encountered particularly in developing countries (1). The prevalence of parasitic infections varies according to the environmental, social and economic factors as well as the age groups (1-3). IPD is known to be more common in children with inadequate hygiene habits compared to the adults. These children are at high risk of iron deficiency anaemia, gastrointestinal disorders and growth retardation, which adversely affect the school success (4).

In our country, there are numerous studies reporting the incidence of childhood IPD varying between $9 \%$ and $60 \%$ and it is more frequent particularly in regions with a low socioeconomic level and poor hygiene and environmental conditions (5-9).

It is important for prevention and treatment of IPD to know the incidence and species of intestinal parasites in the regions. In addition, identification of the associated factors and the attitude and awareness of families about these diseases will play a major role in developing regional plans of preventive measures and determining therapeutic strategies.

To our knowledge, there are no previous relevant studies in the city of Kutahya located in western Turkey. The aim of the present study was to determine the incidence of intestinal parasites and associated factors in primary school students and to assess the knowledge and practices of mothers about these diseases.

\section{MATERIALS AND METHODS}

The study was granted ethical approval by The Medical School Ethics Committee of Eskisehir Osmangazi University and adhered to the Declaration of Helsinki guidelines. Written consent was obtained from the principals of educational institutions included in the study. 


\section{Study Design}

The population of the Kutahya city centre is 237,602 of which 48,317 are children under the age of 15 years. There are 13,563 pupils attaining education at primary schools (age range 5-10 years) in the city center.

The presumable incidence of intestinal parasites was estimated to be $15 \%$ and the study sample was calculated to include 523 pupils to obtain $95 \%$ confidence interval with a $3 \%$ margin of error. In this cross-sectional study, the city centre was divided into 3 regions of a high, moderate and low socioeconomic level according to the data from the Provincial Public Health Directorate. Three schools from each region were selected randomly: Asım Gündüz Primary School (AGPS), Fevzi Cakmak Primary School (FCPS), and Emine Ariogul Primary School (EAPS). Stratified sampling method was used to select the study participants. Stratification was based on the grade the pupils were attending and then on the gender to balance the male to female ratio. The study units were then selected using systematic sampling from each stratum.

The study was carried out between the dates 25/01/2014 and $12 / 03 / 2014$. Parents of the students were invited to the school on a pre-determined day and were informed about the study. Although 538 pupils agreed to participate in the study, only 471 (88\%) had provided the stool sample for laboratory examination. Thus, only the results of these 471 pupils were included in the statistical analysis.

\section{Data Collection}

Study data were collected in two stages. In the first stage, parents were invited to the schools and the pre-prepared questionnaire form and information about the study were handed over. Then, the lidded stool containers and glass slides with cellophane taped on and the information about how to collect a stool sample were given to the participating children. Physical examination of each pupil was completed and, if required, pupils were referred to the hospital for further examination.

The questionnaire form consists of 4 sections. The first part includes the questions about the age, gender and body height and weight of the children, and about disease examination. It inquired about the symptoms of intestinal parasites such as anal itching, salivation from the mouth during sleeping, abdominal pain, and poor weight gain. Pupils with any of these symptoms were considered as having IPD symptoms.

The second part of the survey includes questions about sociodemographic characteristics of parents and the features of residence, while the third part consists of questions of the Personal Hygiene Status Scale (PHSS) developed by the researchers of the present study (10). The PHSS assesses a total of 19 hygiene behaviours including hand washing in a variety of situations, nail cutting, tooth brushing, bathing, changing the underwear, and using wet wipes. Each item is scored on a 5-point Likert scale, with 1 point for "never", 2 points for "rarely", 3 points for "sometimes", 4 points for "mostly", and 5 points for "always". The possible minimum and maximum total scores are 19 and 95 , respectively. The individuals with a score of 81.5 or over are considered to have an adequate personal hygiene.

The fourth part of the survey consists of 17 questions assessing the knowledge level of mothers about intestinal diseases. These questions were aimed at transmission routes and risk factors of intestinal parasitic infestation, preventive measures and treatment of parasitic diseases. Each question is scored as right or wrong with 1 point for the "right". Thus, total score ranges between 0 and 17. This part of the survey also assesses the ethnomedicine/ folk medicine/traditional treatment methods used by the mother to treat IPD.

BMI was calculated from the body height and weight of the participants and was evaluated with Z-score charts based on BMI recommendations from the WHO for males and females aged 5-19 years. Individuals below -2 standard deviations (SD) from standard charts were classified as very thin, individuals ranging from $-2 \mathrm{SD}$ to $-1 \mathrm{SD}$ were classified as thin, individuals ranging from $-1 \mathrm{SD}$ to $+1 \mathrm{SD}$ were classified as normal, individuals between $+1 \mathrm{SD}$ and $+2 \mathrm{SD}$ were classified as overweight, and individuals above +2 SD were classified as obese (11).

The second stage of the study included the collection of stool samples from the pupils and their laboratory analyses in Evliya Celebi Education and Research Hospital Microbiology Laboratory.

\section{Intestinal Parasitic Examination}

Stool samples of $0.5-1.5 \mathrm{~g}$ in lidded and labeled plastic containers were transferred to the laboratory within 1 hour. The preparations from these stool samples were evaluated by native iodine and formaldehyde-ether sedimentation method and the suspicious preparations by staining with trichrom stain. Glass slides with cellophane were examined by microscopy for the presence of eggs of the parasite.

\section{Statistical Analysis}

Data were analyzed using SPSS version 20 statistical package (SPSS, Inc., Chicago, IL, USA). A one-sample KolmogorovSmirnov test was used to assess whether the data were normally distributed. Chi-square analysis was used to compare the frequencies. Logistic regression analysis was used for calculating odds ratio (OR) after data adjustment for age, gender and residence variables. $\mathrm{P}$ values less than 0.05 were considered statistically significant.

\section{RESULTS}

Of the 471 pupils participating in the study, 237 (51\%) were male and 234 (49\%) were female, with a mean age of $7.91 \pm 1.4$ years ranging from 5-11 years.

Of the participants, 181 (38\%) were from EAPS, 160 (34\%) from FCPS and 130 (28\%) from AGPS; 46\%, 23\% and 21\% were attending the $1-2 \mathrm{nd}, 3 \mathrm{rd}$ and 4 th classes, respectively.

The prevalence of IPD was $18.3 \%(\mathrm{~N}=86)$; it was higher in EAPS, which is located in the region with a lower socioeconomic level compared to other two schools $(\mathrm{p}=0.001)$. The prevalence of IPD was not associated with the classroom, gender, number of siblings, and the use of purified drinking water at home ( $p>0.05)$, while the prevalence of IPD was found to decrease with the higher maternal education level $(p=0.002)$.

According to the z-score optimal values, $6.4 \%$ of the students were "very thin", $14.2 \%$ were "thin", $63.9 \%$ were "normal" and $15.5 \%$ were "overweight”. Importantly, the prevalence of intes- 
tinal parasites was significantly higher in thin students compared to the other students $(\mathrm{p}<0.001)$. Similarly, IPD prevalence was found to be higher in students with inadequate hygiene status compared to those with an adequate hygiene $(\mathrm{p}<0.001)$ (Table 1$)$.

Of the parasites found in the stool analysis, $12.1 \%$ were Enterobius vermicularis, 2.5\% Giardia intestinalis, 2.8\% Blastocystis hominis, and 0.4\% Entamoeba coli and Entamoeba histolytica (Table 2). Stool samples from $81.7 \%$ of children were negative. No mixed infections were found.

After adjusting for age and gender, the prevalence of IPD was found to be 4 times higher in regions with a lower socioeconomic level and 3 times higher in 2nd class pupils. The prevalence of IPD was 7 times higher in "low-weight" students and 4 times higher in students with an inadequate hygiene. There was no difference between the groups using or not using purified drinking water at home $(p=0.987)$. With regard to the maternal education level, IPD prevalence was 4 times higher in students whose mothers completed only primary school and 3 times higher in students whose mothers finished high school (Table 3).

The maternal knowledge level score was $12.01 \pm 4.29$ vs. $13.41 \pm 3.94$ in students with and without IPD, respectively $(t=2.917 ; p=0.004)$. With regard to the methods used to treat the IPD, $23 \%$ of the mothers reported that they use conventional methods including drinking chickpea juice (50\%), eating pumpkin

Table 1. Parasite prevalence according to socio-demographic features

\begin{tabular}{|c|c|c|c|c|c|c|}
\hline \multirow[b]{2}{*}{ Variables } & \multicolumn{3}{|c|}{ Parasite status } & \multirow{2}{*}{$\begin{array}{c}\text { Parasite prevalence } \\
\%\end{array}$} & \multicolumn{2}{|c|}{ Statistical comparison } \\
\hline & $\begin{array}{c}\text { Total } \\
(\mathrm{N}=471)\end{array}$ & $\begin{array}{l}\text { Negative } \\
(\mathrm{N}=385)\end{array}$ & $\begin{array}{l}\text { Pozitive } \\
(\mathrm{N}=86)\end{array}$ & & $X^{2}$ & $p$ \\
\hline \multicolumn{7}{|l|}{ Schools } \\
\hline EAPS & 181 & 131 & 50 & 27.6 & \multirow{3}{*}{18.19} & \multirow{3}{*}{0.001} \\
\hline FCPS & 160 & 137 & 23 & 14.4 & & \\
\hline AGPS & 130 & 117 & 13 & 10.0 & & \\
\hline \multicolumn{7}{|c|}{ Level of education } \\
\hline Grade 1 & 132 & 115 & 17 & 12.9 & \multirow{4}{*}{4.38} & \multirow{4}{*}{0.222} \\
\hline Grade 2 & 132 & 102 & 30 & 22.7 & & \\
\hline Grade 3 & 108 & 88 & 20 & 18.5 & & \\
\hline Grade 4 & 99 & 80 & 19 & 19.2 & & \\
\hline \multicolumn{7}{|l|}{ Gender } \\
\hline Boy & 237 & 190 & 47 & 19.9 & \multirow{2}{*}{0.86} & \multirow{2}{*}{0.351} \\
\hline Girl & 234 & 195 & 39 & 16.6 & & \\
\hline \multicolumn{7}{|l|}{ Z score } \\
\hline-3 to -2 & & 24 & 6 & 20.0 & \multirow{4}{*}{22.08} & \multirow{4}{*}{$<0.001$} \\
\hline-2 to -1 & & 42 & 25 & 37.3 & & \\
\hline-1 to 1 & & 252 & 49 & 16.3 & & \\
\hline 1 to 2 & & 67 & 6 & 8.2 & & \\
\hline \multicolumn{7}{|l|}{ Symptom } \\
\hline No & 230 & 192 & 38 & 16.5 & \multirow{2}{*}{0.90} & \multirow{2}{*}{0.340} \\
\hline Yes & 241 & 193 & 48 & 19.9 & & \\
\hline \multicolumn{7}{|l|}{ PHS } \\
\hline Inadequate & 155 & 106 & 49 & 31.6 & \multirow{2}{*}{27.60} & \multirow{2}{*}{$<0.001$} \\
\hline Adequate & 316 & 279 & 37 & 11.7 & & \\
\hline \multicolumn{7}{|l|}{ Drinking water } \\
\hline Non-purified & 260 & 205 & 55 & 21.2 & \multirow{2}{*}{3.25} & \multirow{2}{*}{0.071} \\
\hline Purified & 211 & 180 & 31 & 14.7 & & \\
\hline \multicolumn{7}{|c|}{ Number of siblings } \\
\hline$\leq 2$ & 303 & 243 & 60 & 19.8 & \multirow{2}{*}{1.35} & \multirow{2}{*}{0.244} \\
\hline$\geq 3$ & 168 & 142 & 26 & 15.5 & & \\
\hline Maternal educ & & & & & & \\
\hline Primary & 350 & 279 & 71 & 20.3 & & \\
\hline Secondary & 63 & 51 & 12 & 19.0 & 7.64 & 0.002 \\
\hline High school & 58 & 55 & 3 & 5.2 & & \\
\hline
\end{tabular}


Table 2. Distribution of intestinal parasites

\begin{tabular}{|l|c|}
\hline Parasites & N (\%) \\
\hline \multicolumn{2}{|l|}{ Helminths } \\
\hline Enterobius vermicularis & $57(12.1)$ \\
\hline Protozoa & $12(2.5)$ \\
\hline Giardia intestinalis & $13(2.8)$ \\
\hline Blastocystis hominis & $2(0.4)$ \\
\hline Entamoeba coli & $2(0.4)$ \\
\hline Entamoeba histolytica/dispar & $86(18.3)$ \\
\hline Total &
\end{tabular}

Table 3. Adjusted odds ratio (OR) of variables affecting parasites prevalence

\begin{tabular}{|c|c|c|c|}
\hline Variables & Adjusted OR & $95 \% \mathrm{Cl}$ & $p$ \\
\hline \multicolumn{4}{|l|}{ Schools } \\
\hline EA & 4.55 & $2.06-10.07$ & 0.001 \\
\hline FC & 1.75 & $0.79-3.88$ & 0.166 \\
\hline$A G$ & 1 & & \\
\hline \multicolumn{4}{|c|}{ Level of education } \\
\hline Grade 1 & 1 & & \\
\hline Grade 2 & 3.38 & $1.59-7.21$ & 0.002 \\
\hline Grade 3 & 1.83 & $0.83-4.02$ & 0.134 \\
\hline Grade 4 & 2.23 & $0.99-5.27$ & 0.062 \\
\hline \multicolumn{4}{|l|}{ Gender } \\
\hline Boy & 1 & & \\
\hline Girl & 1.11 & $0.65-1.89$ & 0.708 \\
\hline \multicolumn{4}{|l|}{ Z score } \\
\hline-3 to -2 & 2.03 & $0.54-7.60$ & 0.291 \\
\hline-2 to -1 & 7.55 & $2.58-12.07$ & $<0.001$ \\
\hline-1 to 1 & 2.35 & $0.89-6.16$ & 0.083 \\
\hline 1 to 2 & 1 & & \\
\hline \multicolumn{4}{|l|}{ Drinking water } \\
\hline Non-purified & 1.01 & $0.57-1.78$ & 0.987 \\
\hline Purified & 1 & & \\
\hline \multicolumn{4}{|l|}{ PHS } \\
\hline Inadequate & 3.99 & $2.34-6.79$ & $<0.001$ \\
\hline Adequate & 1 & & \\
\hline \multicolumn{4}{|c|}{ Maternal education } \\
\hline Primary & 3.97 & $1.81-8.74$ & $<0.001$ \\
\hline Secondary & 2.94 & $1.17-7.41$ & 0.022 \\
\hline High school & 1 & & \\
\hline
\end{tabular}

seed (33\%), using the herbal therapies (11\%), and eating yoghurt (6\%) (Fig. 1).

\section{DISCUSSION}

The present study was carried out to determine the prevalence of IPD and associated factors of their occurrence. The prevalence

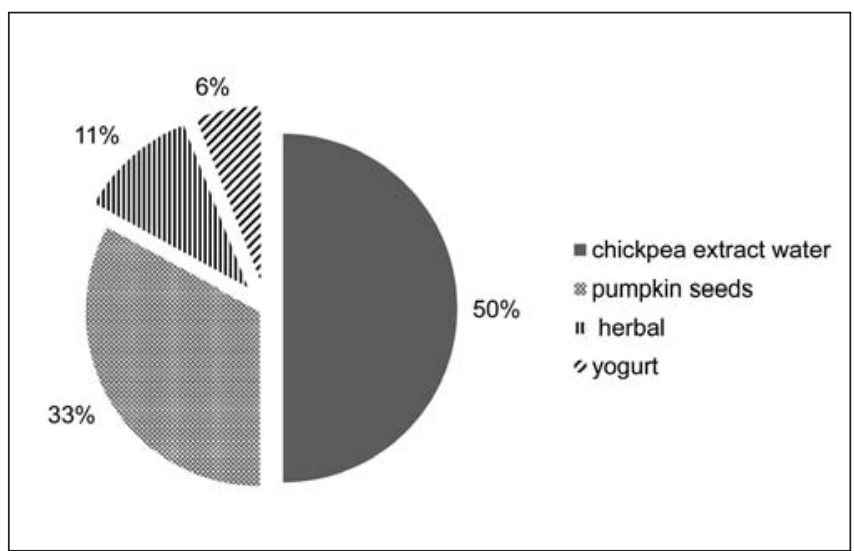

Fig. 1. The traditional methods used by mothers.

of IPD was found to be $18.6 \%$ in primary school pupils in $\mathrm{Ku}-$ tahya, the city located in western Turkey. There are numerous previous studies worldwide on the IPD prevalence in school-aged children. The prevalence of IPD has been reported exceeding 20\% in African countries, 74.7\% in Tanzania, 50.5\% in Rwanda, and 28.1 in Cameroon $(12,13)$.

Several cross-sectional studies carried out in the school-aged children have reported the incidence of IPD to be $22.9 \%$ in China (14), 28.8\% in Iran (15), 86.6\% in Argentina, 55.8\% in Chile and $77.8 \%$ in Peru (16), and the IPD to be endemic in mountainous and rural regions of Cuba (17). The prevalence of IPD has been reported to vary from $9.6 \%$ to $60.4 \%$ in different regions of our country $(5,6,9)$.

Although being lower than that reported in the previous studies, the prevalence of IPD found in the present study is of great importance. In addition, it should be also kept in mind that the climate, environmental conditions and cultural differences are associated with the IPD prevalence (1). The results of our study could be therefore attributed also to the facts that the study was carried out in winter and the schools included in the study were located in the city centre.

In the study by Tagajdid et al. carried out in primary school pupils in an urban region, more than half of the children were found to be infected, which was attributed to the poor hygiene conditions (18). Many previous studies have reported similar results associating the high prevalence of IPD particularly with inadequate hand washing after using the toilet. As a result, the most commonly detected intestinal parasite was Giardia intestinalis $(9,15,19)$. In Libya, it has been reported that $14.6 \%$ of the students aged 5-14 years are infected at least by one intestinal parasite and that most commonly detected intestinal parasites are Giardia intestinalis and Enterobius vermicularis (20).

In the present study, the hygiene status of children was evaluated not only by assessing the hand washing but also by including the general body cleaning as well as by assessing the hygiene status during physical examination. In parallel with the previous studies, about one third (33\%) of the participants had inadequate hygiene and the prevalence of IPS was about 4 times higher in this group of pupils. Moreover, it should be noted that the school toilets and their surroundings are clean and that liquid soap was available at all floors of the schools. We hope that the hygiene-related health educations will be successful as long as these hygiene conditions continue to prevail. 
The most commonly detected intestinal parasite in our study was Enterobious vermicularis (12.1\%), followed by Giardia intestinalis and Blastocystis hominis. The high prevalence of Enterobius vermicularis might result from a possibility that the cellophane tape method for the detection of this parasite was performed correctly by the mothers because during school visits written and practical instructions were given to these mothers about the cellophane tape method and collection of stool samples. Moreover, because the eggs of this parasite contain an embryo, the transmission from the contaminated regions to the non-infected children through the hands is likely, which facilitates the spreading of the disease in crowded environments such as schools attended by children for whom the hygiene habits are not usually adequate.

In a study carried out in 3 suburban Thailand primary schools with different socioeconomic levels, no difference has been found between the schools in terms of IPD prevalence. The authors have also reported that IPD prevalence is not different between male and female pupils but is higher in pupils aged 8 years compared to those aged 7 years (21). Similarly, in a Turkish study, IPD prevalence was not different between the male and female pupils but was higher in the age group of 9 years compared to the age group of 11 years (8). The present study has found relationship between the IPD prevalence and the age and gender. In the first years of the school education, it will take time for the children to establish relationship with friends and to adapt to the school environment. Moreover, the averseness of the 1st class pupils to use the toilet in the school might result in lower prevalence of intestinal parasites in this age group compared to the children attending higher classes. Because the students in 2nd class have more self-confidence but inadequate hygiene habits, the IPD prevalence may be higher in this age group.

In western Turkey, the IPD prevalence has been reported to be higher among individuals living in crowded houses and slum areas with low socioeconomic level and those with uneducated or unemployed parents (9). Many previous studies have showed that the incidence of infections is higher among children of parents with low education level $(15,19,22)$. In the present study, the groups with 3 or more siblings and with a crowded family did not differ in terms of IPD prevalence, while it was found that the prevalence of IPD increases with the decreasing maternal education level. Moreover, in line with the literature, the prevalence of IPD was 4 times higher in the school located in the region with low socioeconomic level (EAPS). Mother is the family member who is primarily responsible for many needs of children including nurturing, nutrition and gaining hygiene habits. Thus, maternal education is very important in order to ensure and maintain all these needs. However, it should be noted that there are many other confounding factors such as the gender of children, family income level or residence.

When parents are aware of the negative impacts of intestinal parasites on the health of their children, they will take measures against the transmission routes of infections and maintain these preventive measures. Thus, it is important for health education activities to determine the target population and to design the related programmes. A previous cross-sectional study in preschool children, maternal knowledge level score has been reported to negatively correlate with the presence of parasitic infections (23). In another study, $41.8 \%$ of the mothers have stated that the source of infection is the soil and foods, while $73 \%$ have stated the importance of preventive measures such as washing hands and 93\% have stressed the role of proper medical treatment (24).

In line with the above-mentioned results, the present study also found lower knowledge level scores among the mothers whose children suffered from IPD. Moreover, of the mothers, 79.6\% stated that IPDs are transmitted through foods and groceries, $86.2 \%$ had pointed out the importance of preventive measures such as washing the hands, 93.2\% stated that these infections should be treated, and about two thirds had adequate knowledge about the disease.

In addition, several applications of the cultural and perceptual norms of the society so called ethnomedicine or folk medicine are also used for the treatment of several diseases. To induce the spilling of the intestinal parasites, commonly called tapeworm, the juice of several vegetables and grains and conventional or herbal treatments are used $(25,26)$. In a study from Turkey, the authors have evaluated the traditional methods used by mothers to treat several childhood diseases and have reported that $12.8 \%$ of the mothers are feeding their children with salt-free pumpkin seeds for the treatment of intestinal parasites (27).

In the present study, $23 \%$ of the mothers stated that they would use folk medicine for IPD treatment, and 50\%, 33\%, 11\% and $6 \%$ stated that they would use chickpea water, pumpkin seeds, herbal cures, and yoghurt, respectively. Because all these traditional methods passed from generation to generation are still used, it is possible that medical treatment may be missed or that health services may be inaccessible. However, further studies are needed to evaluate the effectiveness of these traditional methods in modern medicine.

It has been reported that about $43 \%$ of the pre-school children in developing countries have malnutrition associated with food scarcity and parasitic infections (28). In a research from Ethiopia, the nutritional status of primary school pupils was classified according to their BMI and z-score and the prevalence of intestinal parasites was found to be higher among those with a poor nutritional status (29). Similar results were observed in the present study. The fact that the prevalence of IPD was seven times higher in "low weight" pupils was a significant finding and therefore their nutritional status was investigated, i.e. they were examined by paediatric specialist. To eliminate other reasons of the low weight further physical and laboratory examinations were performed.

\section{CONCLUSION}

In the present study, IPD prevalence was higher among pupils with a lower socioeconomic level, lower maternal education level, malnutrition, inadequate hygiene, and those in the first years of the school-life. The health education programmes about the associated risk factors are of great importance for early detection and treatment of childhood parasitic infections. This will contribute significantly to the physical and mental development of children. Unless being harmful or disrupting the medical treatment, mothers can use traditional methods for the treatment of parasitic infections. Moreover, it will be beneficial for health professionals in terms of patient services to identify and recognize the cultural norms of the society.

We hope that the results of the present cross-sectional study will guide the school health services in the country and the health authorities. 


\section{Ethical Approval}

The study was granted ethical approval by The Medical School Ethics Committee of Eskisehir Osmangazi University - 20.12.2013, no.80558721-77.

\section{Conflict of Interests}

None declared

\section{REFERENCES}

1. Rayan P, Verghese S, McDonnell PA. Geographical location and age affects the incidence of parasitic infestations in school children. Indian J Pathol Microbiol. 2010;53(3):498-502.

2. Rashid M, Rashid S, Rahman A. Prevalence of intestinal parasitoses in urban and rural children of a developing country. Asian Pac J Trop Biomed. 2011;1(2 Suppl):S268-70.

3. Zonta ML, Oyhenart EE, Navone GT. Socio-environmental variables associated with malnutrition and intestinal parasitoses in the child population of Misiones, Argentina. Am J Hum Biol. 2014;26(5):609-16.

4. Alemu A, Atnafu A, Addis Z, Shiferaw Y, Teklu T, Mathewos B, et al. Soil transmitted helminths and Schistosoma mansoni infections among schoolchildren in Zarima town Northwest Ethiopia. BMC Infect Dis. 2011;11:189. doi:10.1186/1471-2334-11-189.

5. Ekinci B, Karacaoğlan E, Bulucu E, Sül N. Investigation of intestinal parasites among elementary school students in the Mugla province. Turkiye Parazitol Derg. 2011;35(2):92-5. (In Turkish.)

6. Güdücüoglu H, Parlak M, Cicek M, Yaman G, Oztürk O, Cikman A, et al. Investigation of intestinal parasites in students of Mustafa Cengiz Primary School in Van. Turkiye Parazitol Derg. 2010;34(3):172-5. (In Turkish.)

7. Calik S, Karaman U, Colak C. Prevalence of microsporidium and other intestinal parasites in children from malatya, Turkey. Indian J Microbiol. 2011;51(3):345-9.

8. Aksoy Ü, Akısü C, Bayram-Delibaş S. Özkoç S, Şahin S, Usluca S Demographic status and prevalence of intestinal parasitic infections in schoolchildren in İzmir, Turkey. Turk J Pediatr. 2007:49(3):278-82.

9. Östan İ, Kilimcioğlu AA, Girginkardeşler N, Özyurt BC, Limoncu ME, Ok ÜZ. Health inequities: lower socio-economic conditions and higher incidences of intestinal parasites. BMC Public Health. 2007;7:342. doi: 10.1186/1471-2458-7-342.

10. Arikan I, Dibeklioglu SE, Arik O, Gulcan A. Personal hygiene status among primary school students in an urban area in the west of Turkey. Am J of Res Commun. 2014;2(7):23-36.

11. World Health Organization [Internet]. Geneva: WHO; c2016 [cited 2013 Nov 21]. Growth reference 5-19 years. Available from: http:www.who. int/growthref/who2007_bmi_for_age/en/index.html.

12. Niyizurugero E, Ndayanze JB, Bernard K. Prevalence of intestinal parasitic infections and associated risk factors among Kigali Institute of Education students in Kigali, Rwanda. Trop Biomed. 2013;30(4):718-26.

13. Barry MA, Weatherhead JE, Hotez PJ, Woc-Colburn L. Childhood parasitic infections endemic to the United States. Pediatr Clin North Am. 2013;60(2):471-85
14. Wang X, Zhang L, Luo R, Wang G, Chen Y, Medina A, et al. Soil-transmitted helminth infections and correlated risk factors in preschool and schoolaged children in rural Southwest China. PLoS One. 2012;7(9):e45939. doi: 10.1371/journal.pone.0045939.

15. Masoumeh R, Farideh T, Mitra S, Heshmatollah T. Intestinal parasitic infection among school children in Golestan province, Iran. Pak J Biol Sci. 2012;15(23):1119-25.

16. Dib J, Oquilla J, Lazarte SG, Gonzalez SN. Parasitic prevalence in a suburban school of Famaillá, Tucumán, Argentina. ISRN Microbiol. 2012:560376. doi: 10.5402/2012/560376.

17. Rojas CL, Angel Núñez CF, Aguiar PH, Silva Ayçaguer CL, Alvarez D, Martínez R, et al. Second national survey of intestinal parasitic infections in Cuba, 2009. Rev Cubana Med Trop. 2012;64(1):15-21. (In Spanish.)

18. Tagajdid R, Lemkhente Z, Errami M, El Mellouki W, Lmimouni B. Prevalence of intestinal parasitic infections in Moroccan urban primary school students. Bull Soc Pathol Exot. 2012;105(1):40-5. (In French.)

19. Wani SA, Ahmad F, Zargar SA, Ahmad Z, Ahmad P, Tak H. Prevalence of intestinal parasites and associated risk factors among schoolchildren in Srinagar City, Kashmir, India. J Parasitol. 2007;93(6):1541-3.

20. Ben Musa N, Sehari A, Hawas A. Intestinal parasitic infections among school children in Tripoli, Libya. J Egypt Soc Parasitol. 2007;37(3):10116

21. Kitvatanachai S, Rhongbutsri P. Intestinal parasitic infections in suburban government schools, Lak Hok subdistrict, Muang Pathum Thani, Thailand. Asian Pac J Trop Med. 2013;6(9):699-702.

22. Shakya B, Shrestha S, Madhikarmi NL, Adhikari R. Intestinal parasitic infection among school children. J Nepal Health Res Counc. 2012;10(1):203.

23. El Sahn AA, Hassan MH, Ftohy EM, Abou-El Ela NE, Eassa SM. Parasitic infections and maternal awareness of preschool children in Karmouz district, Alexandria. J Egypt Public Health Assoc. 2000;75(1-2):1-29.

24. Curtale F, Pezzotti P, Sharbini AL, al Maadat H, Ingrosso P, Saad YS, et al. Knowledge, perceptions and behaviour of mothers toward intestinal helminths in Upper Egypt: implications for control. Health Policy Plan. 1998;13(4):423-32.

25. Quinlan MB, Quinlan RJ, Nolan JM. Ethnophysiology and herbal treatments of intestinal worms in Dominica, West Indies. J Ethnopharmacol. 2002;80:75-83.

26. Giove Nakazawa RA. Traditional medicine in the treatment of enteroparasitosis. Rev Gastroenterol Peru. 1996;16(3):197-202. (In Spanish.)

27. Rahman S, Altan S, Cam S, Yüksel H. Local applications about the child care in Karaağaçlı District of Manisa. Lokman Hekim Journal. 2013;3(1):15-33. (In Turkish.)

28. Tappeh KH, Mohammadzadeh H, Rahim RN, Barazesh A, Khashaveh Sh, Taherkhani H. Prevalence of intestinal parasitic infections among mentally disabled children and adults of Urmia, Iran. Iran J Parasitol. 2010;5(2):60-4.

29. Amare B, Ali J, Moges B, Yismaw G, Belyhun Y, Gebretsadik S, et al. Nutritional status, intestinal parasite infection and allergy among school children in northwest Ethiopia. BMC Pediatr. 2013;13:7. doi: 10.1186/1471-2431-13-7. 